\title{
The recalcitrance of clofibric acid to microbial degradation
}

\author{
S. Evangelista, V. Yargeau \& D. G. Cooper \\ Department of Chemical Engineering, McGill University, Canada
}

\begin{abstract}
The presence of pharmaceutical compounds and their metabolites in aquatic systems has become a concern in the past few years due, in part, to their ubiquity in the environment. However, at present, the persistence and ecotoxicity of many of these compounds remains unknown.

Clofibric acid is the active metabolite of clofibrate, a lipid regulator. It is detected in most aquatic systems where pharmaceutical contaminants are monitored and is reported to be persistent. (R)-mecoprop, a herbicide used on broad-leaved crops, is a structural isomer of clofibric acid. However, unlike clofibric acid, it can be degraded by at least some microorganisms including Sphingomonas herbicidovorans and Alcaligenes denitrificans.

Biodegradation studies of both clofibric acid and its isomer mecoprop were performed by exposing the compounds to axenic cultures of microorganisms in shake flasks. While preliminary results revealed that clofibric acid was resistant to microbial degradation with several types of microorganisms, it was shown that Rhodococcus rhodochrous, a common soil microorganism, was capable of converting clofibric acid to clofibrate, its parent compound. Despite the fact that Sphingomonas herbicidovorans is capable of degrading (R)-mecoprop within a couple of days, results indicated that it is incapable of degrading clofibric acid under the same conditions. This suggests that the recalcitrance of clofibric acid is due to change in the position of a methyl group with respect to (R)-mecoprop.
\end{abstract}

Keywords: clofibric acid, (R)-mecoprop, Sphingomonas herbicidovorans, Rhodococcus rhodochrous, biodegradation. 


\section{Introduction}

The aquatic environment has long acted as a sink for many persistent compounds [1]. Pharmaceutical compounds and their metabolites present in the aquatic environment have begun to receive attention due to their widespread occurrence. Currently, a myriad of pharmaceuticals can be detected in bodies of water across the world, yet their long-term fate in the environment is largely unknown.

Microorganisms are an intrinsic part of the aquatic environment and, thus, play a key role in the degradation and sorption of pharmaceutical contaminants. Studies of the interactions between a compound and microorganisms can impart valuable insight into the environmental fate of the compound and its metabolites.

\subsection{Clofibric acid}

Clofibric acid (Figure 1) is the active metabolite of clofibrate, a ubiquitous lipid regulator. Clofibrate was one of the first commercially available phenoxy compounds to be used as a lipid regulator and was also the first pharmaceutical to be discovered in the aquatic environment in the 1970's due to its similarity to common herbicides [2]. Hitherto, biodegradation experiments have reported that clofibric acid is not readily degraded by microorganisms [3-5]. This strong resistance to biodegradation explains its lengthy environmental persistence which is estimated to be greater than 21 years [6].<smiles>CC(C)(Oc1ccc(Cl)cc1)C(=O)O</smiles>

Figure 1: Chemical structure of clofibric acid.

\section{2 (R)-mecoprop}

(R)-mecoprop (Figure 2) belongs to a group of phenoxy herbicides used to control broad-leaved weeds in cereal crops. It is a structural isomer of clofibric acid, differing only in the position of one methyl group. Various types of microorganisms including Sphingomonas herbicidovorans are known to be able to degrade (R)-mecoprop via cleavage of the ether bond [7]. Metabolites in the biodegradation process include 4-chloro-2-methylphenol and pyruvate [7].<smiles>Cc1cc(Cl)ccc1O[C@@H](C)C(=O)O</smiles>

Figure 2: $\quad$ Chemical structure of (R)-mecoprop. 


\section{Material and methods}

\subsection{Chemicals}

Clofibric acid (2-(4-chlorophenoxy)-2-methylpropanoic acid) and (R)-mecoprop $((\mathrm{R})-(+)-2(2-m e t h y l-4-c h l o r o p h e n o x y)$ propionic acid) and clofibrate were obtained from Sigma-Aldrich Canada Ltd.

\subsection{Cultures}

Microorganisms were first grown in their optimal media as recommended by ATCC. Only axenic cultures were used in the trials. When stationary phase was attained, a $1 \mathrm{ml}$ sample was transferred to a $500 \mathrm{ml}$ shake flask containing 100 $\mathrm{ml}$ of minimal mineral salts media, yeast extract $(0.1 \mathrm{~g} / \mathrm{l})$, glucose $(2.5 \mathrm{~g} / \mathrm{l})$ and clofibric acid $(0.1 \mathrm{~g} / \mathrm{l})$. The clofibric acid was added to the shake flasks prior to autoclaving. The cultures were kept at $26^{\circ} \mathrm{C}$ in an incubator shaker at $150 \mathrm{rpm}$. For the (R)-mecoprop trials, an analogous procedure was used. The length of the trials was dependent on the rate of degradation of the compound in study.

\subsection{Sample analysis}

Samples were extracted using chloroform containing benzoic acid $(1 \mathrm{~g} / \mathrm{l})$ as internal standard, in a 1:2 (solvent:broth) volume ratio. Extracted samples were analysed using gas chromatography.

The analysis was performed using a Varian GC equipped with a flame ionization detector (FID).

For metabolite identification, analytical standards obtained from SigmaAldrich Canada Ltd were used in the GC. Further studies were done with GCmass spectroscopy.

\section{Results}

A number of organisms were tested for the ability to degrade clofibric acid (Table 1). Some data for (R)-mecoprop are also included in this table.

Table 1: Microorganisms screened in biodegradation experiments.

\begin{tabular}{cccc}
\hline & & \multicolumn{2}{c}{ Degradation observed } \\
\hline Microorganism & ATCC & Clofibric acid & Mecoprop \\
\hline Pseudomonas putida & 12633 & - & Not tested \\
Aspergillus niger & 16888 & - & Not tested \\
Bacillus subtilis & 6051 & - & Not tested \\
Pseudomonas fluorescens & 13525 & - & Not tested \\
Spingomonas herbicidovorans & 700291 & - & + \\
Rhodococcus rhodochrous & 13808 & + & - \\
\hline
\end{tabular}


None of the organisms tested were capable of degrading clofibric acid. Rhodococcus rhodochrous did appear to cause a loss of clofibric acid but this missing material was merely its ethyl ester, attaining almost a complete conversion after 20 days of inoculation (Figure 3). Remarkably this is the actual form of the pharmaceutical, which is converted to clofibric acid after ingestion. This phenomenon was observed after the bacteria had been exposed to clofibric acid in previous trials.

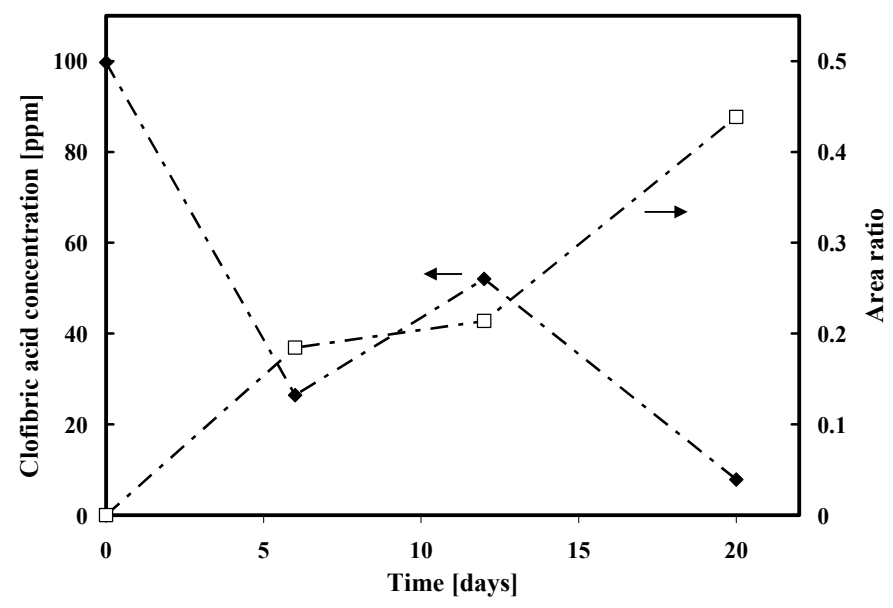

Figure 3: $\quad$ Conversion of clofibric acid ( $\bullet$ ) to clofibrate ( $\square$ ) by Rhodococcus rhodochrous.

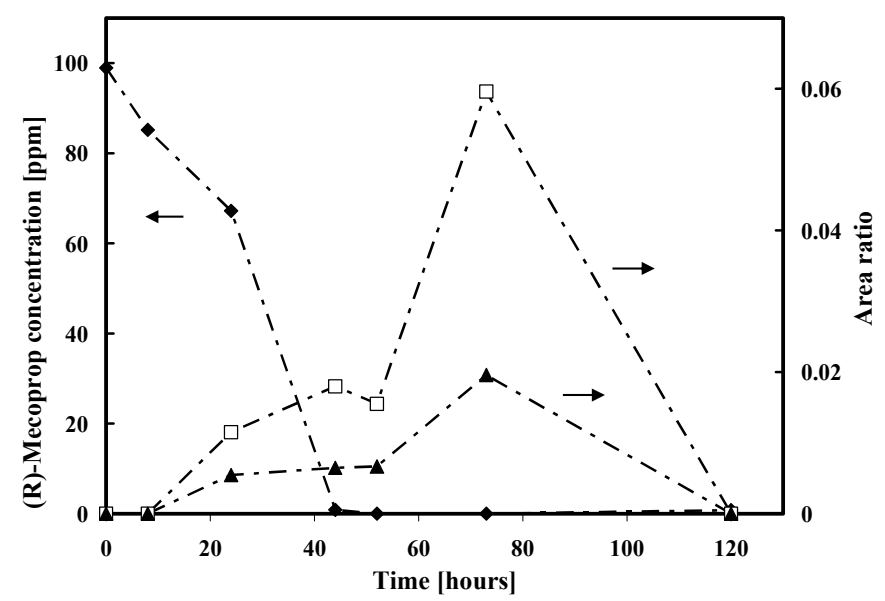

Figure 4: Degradation of (R)-mecoprop ( $\bullet$ by Sphingomonas herbicidovorans. Appearance of two metabolites $(\boldsymbol{\Lambda}),(\square)$ during degradation. 
Despite the fact that Sphingomonas herbicidovorans was capable of degrading (R)-mecoprop, results indicated that it was incapable of degrading its isomer, clofibric acid. The complete degradation of (R)-mecoprop by Sphingomonas herbicidovorans occurred within two days, and two transient metabolites were seen as degradation proceeded (Figure 4). Investigation into the identity of these two metabolites is necessary to confirm the same degradation pathway referenced in the literature.

\section{Discussion}

The structure of clofibric acid is crucial to its lack of biodegradability. The first step in the biodegradation of its isomer, (R)-mecoprop, is cleavage of the ether bond [7]. In general, an ether bond is known to confer a high degree of resistance in the biological mineralization of xenobiotic compounds [8]. Moreover, the chlorophenoxy herbicides, to which (R)-mecoprop can be assigned, are known to be slowly biodegraded and only by a limited number of microorganisms which are known to possess enzymatic systems capable of ether bond cleavage [8].

Sphingomonas herbicidovorans is one of these organisms that can degrade (R)mecoprop but is ineffective in degrading clofibric acid. This suggests that the extra methyl group on the propionic acid $\alpha$ to the ether group creates the recalcitrance of clofibric acid due to steric hindrance.

Rhodococcus rhodochrous, although unable to degrade clofibric acid, can convert it into the ethyl ester clofibrate, decreasing its solubility in the aqueous media. However, conversion from clofibric acid into clofibrate was only seen after the bacteria had been exposed to clofibric acid in previous trials. When the bacteria had not been in any way acclimated to clofibric acid, then the same result was not seen. This suggests that microbial acclimation to clofibric acid had occurred.

\section{Conclusion}

Clofibric acid is a very recalcitrant compound and is not readily degraded by microorganisms. The two methyl groups on the carbon adjacent to the ether bond appear to be the cause of this lack of biodegradability. (R)-mecoprop, a structural isomer of clofibric acid with only a single methyl group at this position, can be degraded by bacteria known to have the enzymes capable of hydrolyzing an ether bond.

\section{Acknowledgements}

This work was made possible due to the support from the Natural Sciences and Engineering Research Council of Canada, the Fonds Québécois de la Recherche sur la Nature et les Technologies (FQRNT) and the Eugenie Ulmer Lamothe fund of McGill University. 


\section{References}

[1] Buser, H.-R., M.D. Müller, and N. Theobald, Occurrence of the pharmaceutical drug clofibric acid and the herbicide mecoprop in various Swiss lakes and in the North Sea. Environmental Science \& Technology, 32 (1): p. 188-192, 1998.

[2] Kümmerer, K., Pharmaceuticals in the Environment: Sources, Fate, Effects and Risks, ed. S.-V.B.H.N. York. 2001.

[3] Zwiener, C., T. Glauner, and F.H. Frimmel, Biodegradation of pharmaceutical residues investigated by SPE-GC/ITD-MS and on-line derivatization. Hrc-Journal of High Resolution Chromatography, 23 (7-8): p. 474-478, 2000.

[4] Zwiener, C. and F.H. Frimmel, Short-term tests with a pilot sewage plant and biofilm reactors for the biological degradation of the pharmaceutical compounds clofibric acid, ibuprofen, and diclofenac. Science of the Total Environment, 309(1-3): p. 201-211, 2003.

[5] Winkler, M., J.R. Lawrence, and T.R. Neu, Selective degradation of ibuprofen and clofibric acid in two model river biofilm systems. Water Research, 35(13): p. 3197-3205, 2001.

[6] Emblidge, J.P. and M.E. DeLorenzo, Preliminary risk assessment of the lipid-regulating pharmaceutical clofibric acid, for three estuarine species. Environmental Research, 100(2): p. 216-226, 2006.

[7] Nickel, K., M.J. Suter, and H.P. Kohler, Involvement of two alphaketoglutarate-dependent dioxygenases in enantioselective degradation of (R)- and (S)-mecoprop by Sphingomonas herbicidovorans $M H$. Journal of Bacteriology, 179(21): p. 6674-6679, 1997.

[8] White, G.F., N.J. Russell, and E.C. Tidswell, Bacterial scission of ether bonds, Microbiological Reviews, 60(1), p. 216-232, 1996. 